\title{
Erratum: Implementing universal nonadiabatic holonomic quantum gates with transmons [Phys. Rev. A 97, 022332 (2018)]
}

Zhuo-Ping Hong, Bao-Jie Liu, Jia-Qi Cai, Xin-Ding Zhang, Yong Hu, Z. D. Wang, and Zheng-Yuan Xue

(Received 10 May 2018; published 23 May 2018)

DOI: 10.1103/PhysRevA.97.059905

In the original paper there are typographical errors that should be corrected as listed in the following.

In the main text, the angle in Fig. 1(b) should be $\gamma$ as indicated in Eq. (2). The corrected figure is now shown in Fig. 1. Meanwhile, on p. 4, below Eq. (7), $F_{2}=99.44 \%$. The original fidelity $99.27 \%$, which contains nonexisting decoherence, was used by mistake.

In Appendix A, on the first line of p. 5, the corrected form of a transmon's lower operator should be $b=|g\rangle\langle e|+\sqrt{2}| e\rangle\langle f|+$ $\sqrt{3}|f\rangle\langle h|+\cdots$. Meanwhile, the definition of $\alpha$ in Appendix A and the main text has opposite signs, and thus the first lines of Eqs. (A1), (A2), and (A9) should read

$$
\begin{aligned}
H_{0} & =\delta_{r} n_{a}+\delta_{q} n_{b}-\frac{\alpha}{2}\left(n_{b}-1\right) n_{b}, \\
E_{f, 0} & =2 \delta_{q}-\alpha, \quad E_{g, 1}=\delta_{r},
\end{aligned}
$$

and

$$
\begin{aligned}
\eta_{g 1} & =\frac{g^{2}}{\Delta}-\frac{\Omega^{2}}{4(\Delta+\alpha)} \\
\eta_{f 0} & =\frac{\Omega^{2}}{2 \Delta}-\frac{2 g^{2}}{\Delta+\alpha}-\frac{3 \Omega^{2}}{4(\Delta-\alpha)} \\
\tilde{g} & =\frac{\sqrt{2} g \Omega e^{-i \phi}}{2 \Delta}-\frac{\sqrt{2} g \Omega e^{-i \phi}}{2(\Delta+\alpha)} \\
& =\frac{g \Omega e^{-i \phi} \alpha}{\sqrt{2} \Delta(\Delta+\alpha)} .
\end{aligned}
$$

(a)

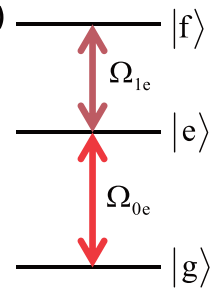

(d)

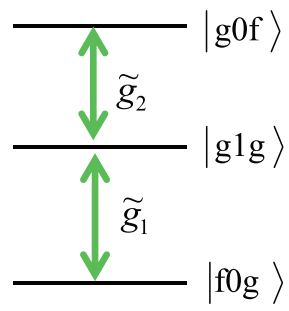

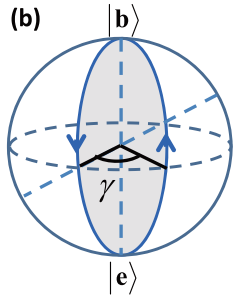

(c)

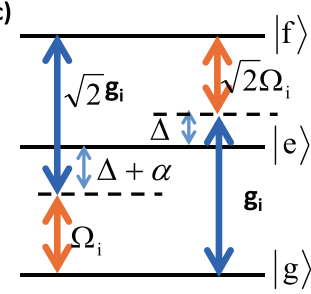

(e)

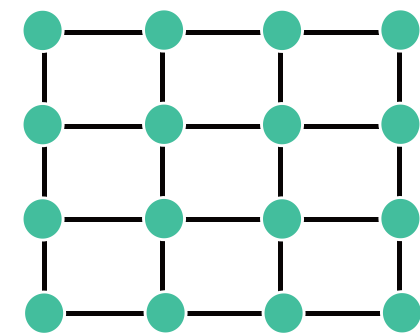

FIG. 1. Corrected panel (b). 
In addition, the inline formula between Eqs. (A2) and (A3) should be corrected as $\delta_{r}=2 \delta_{q}-\alpha$. Finally, in Appendix B, the last unnumbered equation should read

$$
\left\langle\phi_{j}(\Omega)\left|\frac{\partial H}{\partial \Omega}\right| \phi_{i}(\Omega)\right\rangle+\frac{d \omega}{d \Omega}\left\langle\phi_{j}(\Omega)\left|\frac{\partial H}{\partial \omega}\right| \phi_{i}(\Omega)\right\rangle=0 .
$$

All the corrected forms of the equations are actually used in our calculations. Therefore, these typographical errors do not affect any other results of the paper. 